\title{
The Design of multi-purpose industrial timer warning system
}

\author{
Ying Wang ${ }^{1, \text { a }}$ Yang yang $\mathrm{Xu}{ }^{1, \mathrm{~b}}$ \\ Zhengzhou University of Industry Technology, Zhengzhou 450007, China. \\ aWin0725@163.com, b xuyangyang0120@126.com
}

Keywords: Timer, Relative Timing, Absolute Timing,STC89C51.

\begin{abstract}
This paper mainly designs a multi-purpose industrial timer that based on STC89C51.The multi-purpose industrial timer uses the calendar chip DS1302, LCD TS1620, buzzers and other devices. The design uses a $\mathrm{C}$ language programming, including liquid crystal display program, calendar program, key scanner. The industrial timer should be able to achieve absolute time and relative time of the time, a wide range of time, and a signal output.
\end{abstract}

\section{Introduction}

Application of the timer is more and more widely, It Often used in industry for the time delay electronic timer shutdown, timer, alarm automatically. Mechanical timers are used in industrial production is designed according to the principle of general wind-up clocks and watches, although this timer has simple structure, low cost, maintenance is convenient, but its frequent contact and contact disconnect, greatly reduced its service life, and It does not conducive to further fully automated. Advantages of electronic timer become more obvious, It will be gradually replaced by electronic timer, this is self-evident. This paper mainly discussed from the following several aspects:

\section{The overall scheme design}

This design mainly includes the hardware design and software design part, Power supply circuit module, keys module, STC8951 MCU and clock chip DS1302, buzzer, LCD TS1620. Design block diagram as shown in figure 1.

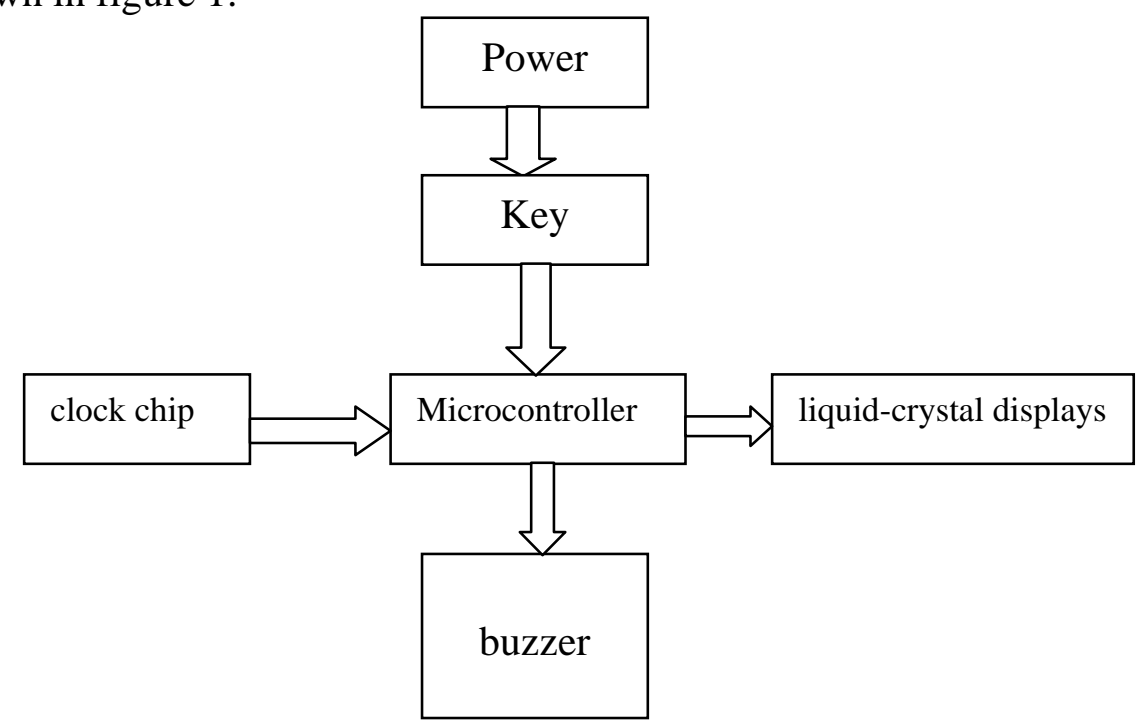

Fig.1 System overall scheme

\section{The hardware circuit design}

The hardware circuit is mainly composed of the following aspects: STC89C51 SCM control module, keys module, clock chip DS1302 module, liquid crystal display TS1620 module, buzzer module, etc. 


\section{Single-chip microcomputer control module .}

STC89C51 is with a $4 \mathrm{k}$ bytes of scintillation programmable and can erase read-only memory type low voltage, high performance CMOS8 bit microprocessors, commonly known as single chip microcomputer. The device adopts the high-density nonvolatile storage manufacture technology, and industry standard of MCS-51 instruction set and output tube compatible [2]. In the single chip microcomputer control module mainly includes the following several control part. (1) The clock circuit. The external clock. External clock pulse received XTAL1 side, namely the input end of the internal clock generator, XTAL2 are hung up. (2) reset circuit.

\section{The power supply module}

The power supply circuit using 7805 voltage chip, thus ensuring the $+5 \mathrm{v}$ voltage output [3]. Figure in the diode D1 in order to prevent the reverse, a longer C2 is used to offset the input connection induction, prevent self-excited oscillation. C1 to the instantaneous load current increase or decrease when not cause output have larger fluctuation, C1 and C2 can choose between $0.1 \sim 100$ micro method commonly. Power supply circuit module diagram is shown in figure 2.

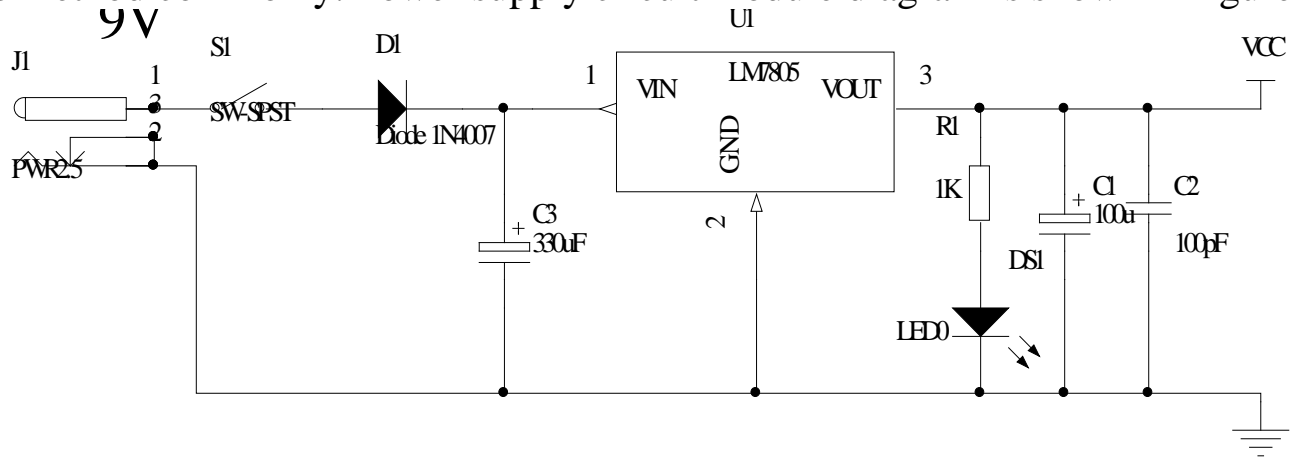

Fig.2 Power circuit

\section{The clock chip}

This design using DS1302, it is by DALLAS company launched a trickle charging the clock chip, contains a real-time clock/calendar and 31 bytes static RAM, Through a simple serial interface communicate with MCU [4]. Real time clock/calendar circuit with second points, the hour, the day, date, month and year of information, The number of days per month and leap year the number of days can be automatically adjusted, clock operation can decide it directed by an AM/PM 12 or 24 hour format, DS1302 and MCU can simply adopt the way of synchronous serial communication.

\section{The software design}

Software program mainly includes the main program, read buttons, LCD display program, perpetual calendar, speaking, reading and writing program and the buzzer rang, etc.The overall software design framework is shown in figure 3.

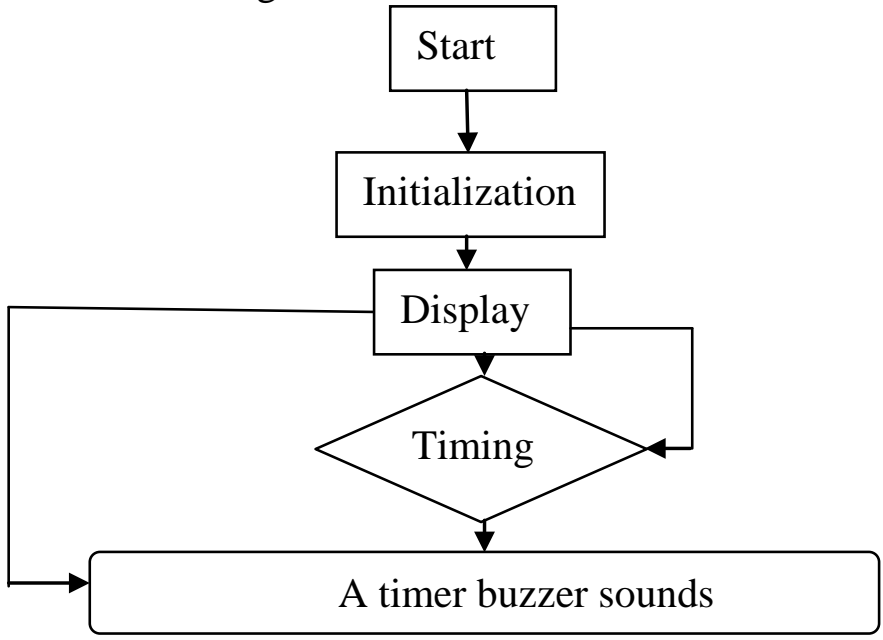

Fig.3 System software design framework 


\section{Key reading program}

Read the key procedures in the timer, 50ms scan a keyboard, the program has to shake function, avoids the external misoperation caused by interference signals. The key function to specify:

Keys K1: when regulation, each click this button, the time of a plus one. For example: when the tuning time in points, click this button to add one.

Keys K2: when regulation, each click this button, the time of a minus one. For example: when the tuning time in, press this button can reduce a.

Keys K3: when regulation, each click this button, when the time in timing or adjustable, the adjustable time of. For example, when the time is in second place, push down this button, who will jump to the tuning time points.

Keys K4: when regulation, each click this button, when the time in the timing or debug, side or tune time can determine the time. For example, when the set time, push down this button indicates time finished, the timer will perform this time.

Keys K5: a function to achieve the tuning of the timer, or switch between absolute time, with respect to time. For example: when the time is normal, push down this button, then enter the absolute time, press again, side into the absolute time.

First determine whether K5 keys press, It will not on time if it not press is. If pressed into the pitch time state, click into the state, according to two times, enter a state of absolute timing, according to the three times has entered into a state of relative timing.After judge whether button K4 press, press is to determine the current state, after judge whether K3 button press, press, select the indicated time, After the judgment of $\mathrm{K} 1$ and $\mathrm{K} 2, \mathrm{~K} 1$ press said to increase the time, $\mathrm{K} 2$ press is said to reduce time.Finally determine whether button K4 press down, if not press, can continue to set the tone.If the said end of debugging, into a state timer timing, timing function.

\section{Display program}

LCDTS1620 display program including LCDTS1620 initialization, read/write operations, and the related characters, pictures and other code call subroutine.On the LCD screen do read and write operations, before writing, should be paid attention to drop fails to cover the content of the qing dynasty, otherwise, the accumulative effect in characters, not very clear.Liquid crystal display (LCD) flow chart as shown in figure 4.

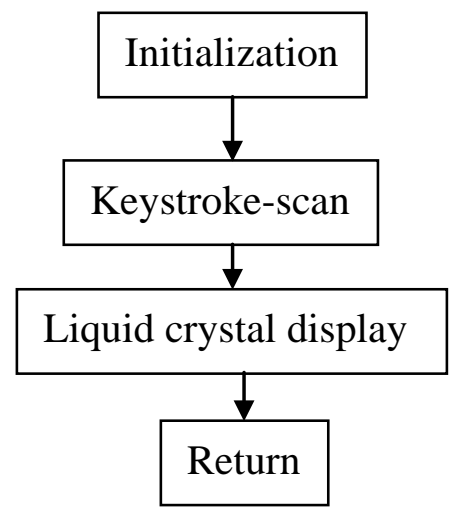

Fig.4 Flow chart of liquid crystal display (LCD)

\section{Time reading program}

From time chip DS1302 read time (year, month, day, week,, minutes and seconds), also need to strictly abide by the temporal logic (single bus structure) and read if you want to display the time, also must pass a number system conversion, BCD code is converted to a decimal) [5].

\section{System debugging}

To combine with the above procedure, the system hardware circuit to connect again good, after the overall program downloaded to the MCU, after turning on the power supply, found that is not all, liquid crystal display in the part of knowledge need to display, then look again at LCD specifications, found that after each line of liquid crystal display (LCD) is only 16, change the program again, make 
year shows a take out, then to show to the premise two, so finally achieved the design requirements of all functions can realize absolute timing and relative timing of industrial, can satisfy the timing requirements of most industrial [6].

\section{Summary}

The practical timer designed in this paper has the advantages of small volume and integral design. The meter is delicate, easy to operate, low cost, strong function and time range Wide, high precision, wide range of uses and other features, is an ideal timing Tools, after several trials and improvements are now in the user trial, reflecting the effectiveness of Fruit good. The timer can be quickly translated into actual products, market prospects Broad, expected economic efficiency is higher. At the same time, The industrial timer can be able to achieve absolute time and relative time of the time, a wide range of time, and a signal output.

\section{References}

[1] .Shao ming Xu, Jiang hai $\mathrm{Xu}$,Wei hua Shi. Design and Realization of Practical Timer Based on AT89C51.Journal of Huaihai Institute of Technology(Natural Science Edition)Vol. 21 (2012) No. 04, p. 37-40.

[2]. Xu guang Zhu. Application of MCU's timer. Techniques of Automation and Applications. Vol. 31 (2012) No. 01, p. 99-103.

[3]. Lei Yang, Yu fu Sheng, Mei jing Guo. AT89S52 based timer design. Journal of Shandong Jianzhu University. Vol. 21 (2006) No. 05, p. 463-470. 